\title{
RIQUEZA E COMPOSIÇÃO DE ESPÉCIES DE FORMIGAS NO PROCESSO DE RECUPERAÇÃO DE UMA VOÇOROCA
}

\author{
Gabriel Biagiotti ${ }^{1 *}$, José Aldo Alves Pereira², Carla Rodrigues Ribas², Vanesca Korasaki², \\ Ronald Zanetti², Antônio César Medeiros de Queiroz ${ }^{2}$
}

*Autor para correspondência: gbiagiotti@yahoo.com.br

RESUMO: Neste trabalho, objetivou-se verificar como a riqueza e a composição de espécies de formigas se comporta com as mudanças ocorridas no processo de recuperação de uma voçoroca. A área em estudo possui 0,9 hectares subdivididos em três setores denominados braços: "A"; "B" e "C". Para a definição dos setores tomou-se como base o nível de atividade erosiva e a regeneração natural. Foram lançadas quatro transeções de forma sistemática na área da voçoroca e no entorno composto com mata e pastagem. Cada transeção possuía três armadilhas do tipo "pitfall" distante entre si 10 metros, sendo que as capturas de formigas foram realizadas em duas épocas, chuvosa e seca. Foi realizada uma análise de variância para comparar o número de espécies de formigas capturadas por parcela nos ambientes e aplicado teste Scott-Knott a 5\% para comparação das médias. Para verificar a similaridade de espécies entre os ambientes foi realizada uma análise de similaridade (ANOSIM) e para a ordenação dos ambientes uma "Nonmetric Multidimensional Scaling" (NMDS). Foram capturadas 74 espécies de formigas no interior da voçoroca e no entorno. O ambiente mais degradado e em estágio inicial de regeneração, apresentou maior riqueza de espécies de formigas. A composição de espécies de formigas foi diferente entre os ambientes em recuperação e o entorno. Os parâmetros das comunidades de formigas analisados, riqueza e composição de espécies, foram influenciados pela regeneração da área, indicando que as formigas podem ser usadas como bioindicadoras de recuperação de voçorocas.

Palavras-chave: Áreas degradadas, monitoramento ambiental, formicidae.

\section{RICHNESS AND SPECIES COMPOSITION OF ANTS IN THE RECOVERY PROCESS OF A GULLY EROSION}

\begin{abstract}
This study aimed to determine how the richness and composition of ant species behaves with changes in the recovery process of a gully erosion. The study area has 0.9 hectares subdivided into three sections called sector: " $A$ ", " $B$ " and " $C$ ". For the definition of the sectors, erosive and natural restoring were taken as the base level of activity. Four transects were laid systematically throughout the area and surrounding compound with forest and grassland. Each transect had three "pitfall trap" ten meters apart from each other, with catches of ants were held in rainy and dry seasons. Analysis of variance was applied to compare the number of ant species per plot captured and Scott-Knott test 5\% for comparison of means. To verify the similarity of species between environments it was performed an analysis of similarity (ANOSIM) and ordering of environments a "Nonmetric Multidimensional Scaling" (NMDS). We captured 74 species of ants inside and around the gully erosion. The more degraded environment and initial stage of regeneration, showed greater richness of ant species. The composition of ant species was different between the recovery environments and around. The parameters of ant communities analyzed, richness and composition species were influenced by the regeneration of the area, indicating that ants can be used as bioindicators of gullies recovery.
\end{abstract}

Key words: Degraded areas, environmental monitoring, formicidae.

\section{INTRODUÇÃO}

Avaliações a partir de respostas biológicas podem identificar indiretamente o grau de perturbação e também o nível de recuperação de uma área degradada. Dessa forma, os bioindicadores são espécies ou grupo de espécies, que de alguma maneira refletem o estado biótico e ou abiótico do ambiente (NIEMI; MCDONALD, 2004).

Dentre as espécies potenciais, os insetos tem-se mostrado bons indicadores de mudanças ambientais (MALEQUE et al., 2009; MCGEOCH, 1998; UEHARA-
PRADO et al., 2009), na medida em que algumas características intrínsecas desses organismos podem detectar mudanças no ambiente, mesmo em uma escala local.

No filo dos artrópodes as formigas representam um grupo com grande potencial de utilização em programas de monitoramento para conservação ambiental (RIBAS et al., 2012; UNDEWOOD; FISHER, 2006). As características que fazem desse grupo bons indicadores são: abundância de indivíduos, ampla distribuição, fácil amostragem e identificação das espécies (ALONSO; AGOSTI, 2000).

${ }^{1}$ Universidade Estadual Paulista "Júlio de Mesquita Filho" - Jaboticabal, São Paulo, Brasil

${ }^{2}$ Universidade Federal de Lavras - Lavras, Minas Gerais, Brasil

Cerne, Lavras, v. 19, n. 4, p. 661-668, out./dez. 2013 
As formigas apresentam importantes relações com as plantas e outros animais (BENNETT et al., 2009), e participam de processos naturais, onde são consideradas dispersoras de sementes (LEAL, 2003), polinizadoras, além de "guarda-costas" de algumas plantas. Participam também da ciclagem de nutrientes na sucessão vegetal e do controle biológico de pragas (HÖLLDOBLER; WILSON, 1990).

No monitoramento e processos de recuperação de áreas degradadas é fundamental o estudo da comunidade das formigas e suas interações, pois elas mantêm e restauram a qualidade do solo, além de operar na redistribuição de partículas do solo e melhorar a infiltração de água pelo aumento da porosidade (BRUYN, 1999).

Este trabalho foi realizado em uma área degradada por erosão hídrica com ocorrência de voçoroca, sob o regime de regeneração natural e, no seu entorno composto por vegetação de cerrado stricto sensu, e área de pastagem plantada. O objetivo foi verificar como a riqueza e a composição de espécies de formigas se comporta no processo de recuperação de uma voçoroca.

\section{MATERIAL E MÉTODOS}

A área em estudo encontra-se no município de Itumirim, nas coordenadas $21^{\circ} 16^{\prime}$ Sul e $44^{\circ} 50^{\prime}$ ' Oeste, na região sul do Estado de Minas Gerais e pertence à bacia hidrográfica do rio Grande. O clima do município é de transição entre Cwa e Cwb, de acordo com a classificação climática de Köppen (ANTUNES, 1986), sendo caracterizado como clima mesotérmico, com inverno seco e verão chuvoso e precipitação média anual de $1.530 \mathrm{~mm}$. A altitude média varia de 900 a $950 \mathrm{~m}$.

As classes de solos predominantes no local são os Latossolos Vermelho-Amarelo e Cambissolos, derivados da alteração do gnaisse-granítico (LOSCHI, 2009). A vegetação original da região era composta por um mosaico de diferentes fitofisionomias de cerrados (CARVALHO, 1992), as quais foram substituídas por pastagens e culturas agrícolas, restando apenas pequenos fragmentos da vegetação.

A voçoroca surgiu em uma propriedade rural particular e possui uma área de 0,9 hectares e formato irregular. Como no seu interior ocorrem situações diferentes de regeneração natural ela foi subdividida em três sítios, denominados: braços "A"; "B" e "C" (Figuras 1 e 2). A definição desses sítios foi realizada com base no estado da atividade erosiva e da regeneração natural.

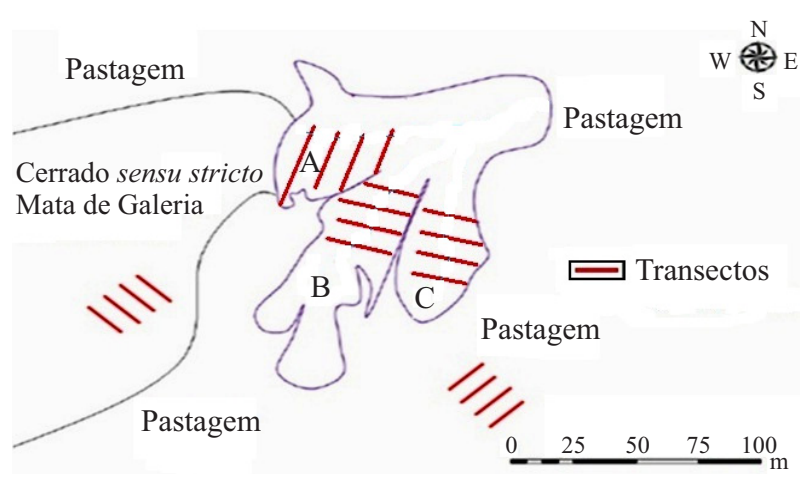

Figura 1 - Caracterização da área estudada mostrando a localização das transeções lineares nos três braços "A", "B", "C" e a matriz de entorno, pastagem e mata, Itumirim-MG, adaptado de Loschi (2009).

Figure 1 - Characterization of study area showing the location of linear transects in the sectors " $A$ ", " $B$ ", " $C$ " and the surrounding matrix, grassland and forest, Itumirim- $M G$, adaptation of Loschi (2009).

O braço "A" (Figuras 1 e 2) é o mais estável quanto à atividade erosiva, encontrando-se em processo avançado de regeneração natural; o braço "B" (Figuras 1 e 2) apresenta-se estabilizado e em estágio intermediário de regeneração natural, e o braço "C" (Figuras 1 e 2), que se encontra em processo ativo de erosão e em estágio inicial de regeneração natural.

As formigas foram amostradas de forma sistemática na área da voçoroca e na matriz de entorno de cerrado stricto sensu, composta por pastagem plantada e mata ciliar, por meio de parcelas alocadas ao longo de quatro transeções lineares por ambiente, distantes 10 metros entre si (Figura 1).

Foram instaladas no total 60 armadilhas, três armadilhas do tipo "pitfall" por transeção. As armadilhas foram feitas com potes redondos de material plástico, com diâmetro de seis centímetros e altura de sete centímetros. No interior de cada pote, colocou-se água salobra com detergente neutro, os quais foram enterrados rente ao solo onde permaneceram no local pelo período de 48 horas em duas épocas de amostragem, uma no verão (época chuvosa) e outra no inverno (época seca).

Após a retirada das armadilhas, as formigas foram triadas com auxílio de lupa estereoscópica e montadas para identificação no Laboratório de Entomologia Florestal do DEN/UFLA. A identificação dos formicídeos foi realizada com auxílio da chave de Bolton (1994) e comparações com coleções de referência.

Cerne, Lavras, v. 19, n. 4, p. 661-668, out./dez. 2013 


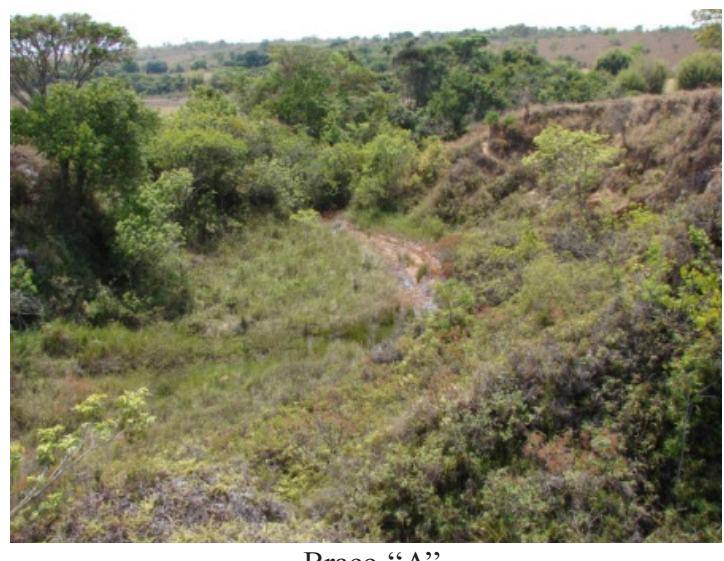

Braço "A"

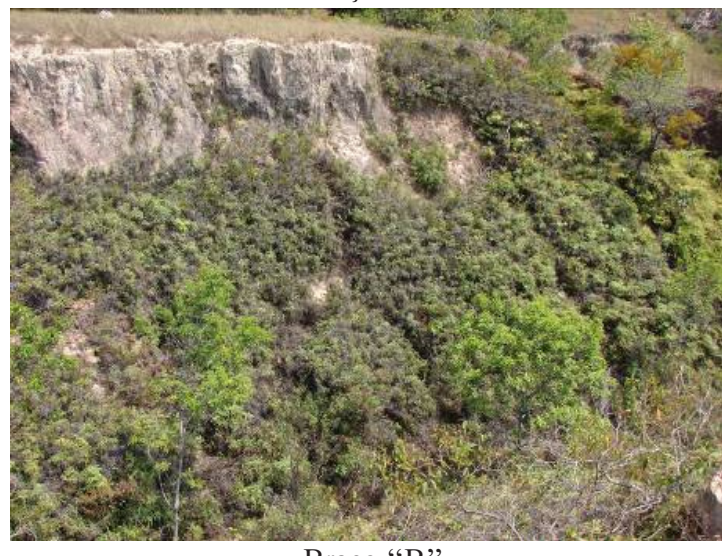

Braço "B"

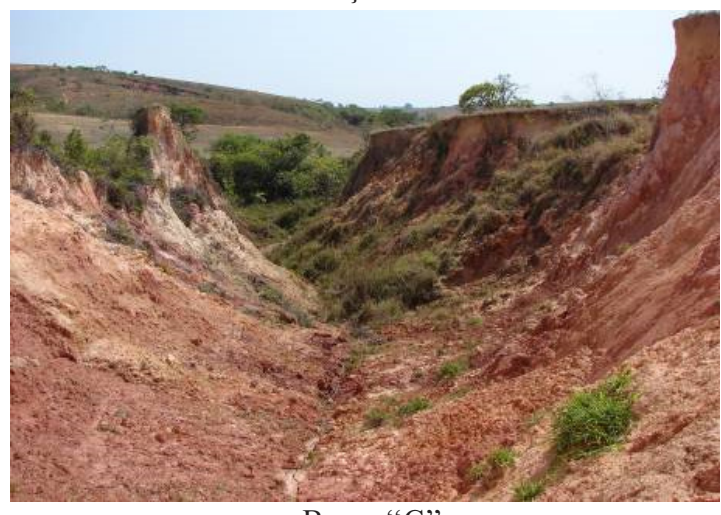

Braço "C"

Figura 2 - Fotos ilustrando ambientes no interior da voçoroca em regeneração (Braços "A": estágio de regeneração avançado, Braço "B": estágio de regeneração intermediário, Braço "C": estágio de regeneração inicial), Itumirim-MG (2010).

Figure 2 - Pictures illustrating environments within the gully erosion in regeneration (Sector " $A$ ": advanced stage of regeneration, Sector " $B$ ": intermediate stage of regeneration, Sector " $C$ : inicial stage of regeneration), Itumirim-MG (2010).
Para verificar a riqueza de formigas nos ambientes em recuperação no interior da voçoroca em comparação com o entorno foi realizada uma análise de variância (ANOVA) das médias do número de espécies de formigas capturadas por "pitfall" aplicando o teste F, e as mesmas foram comparadas pelo teste de Scott-Knott a 5\% de probabilidade. As análises foram realizadas, utilizando-se o software R (R DEVELOPMENT CORE TEAM, 2008).

Para avaliar a composição de espécies de formigas nos diferentes graus de regeneração no interior da voçoroca e do seu entorno foi utilizado uma análise "Nonmetric Multidimensional Scaling" (NMDS) para revelar a ordenação das parcelas diante da composição de espécies de formigas. A ordenação foi feita utilizando-se dados de presença e ausência, empregando-se o índice de similaridade Raup-Crick. As possíveis similaridades na composição de espécies de formigas entre os ambientes no interior da voçoroca e do entorno foram identificadas efetuando o teste de similaridade ANOSIM (CLARKE; WARWICK, 2001) que, por meio de um valor de correlação, nos permite observar diferenças nos ambientes em função da composição de espécies de formigas. As análises NMDS e ANOSIM foram realizadas com o programa Paleontological Statistics PAST (HAMMER et al., 2010).

\section{RESULTADOS E DISCUSSÃO}

No total, foram capturadas 74 espécies de formigas no interior da voçoroca e no entorno, mata e pastagem (Tabela 1).

Existe diferença significativa entre o número de espécies de formigas capturadas nos ambientes da voçoroca e entorno $(F=5,047 ; p<0.01)$. Os ambientes braço " $\mathrm{C}$ " na voçoroca e pastagem apresentaram os maiores número de espécies de formigas capturadas por "pitfall", diferenciando-se estatisticamente dos demais ambientes (Figura 3).

Os dados obtidos neste trabalho sugerem relações não esperadas, pois o ambiente braço " $\mathrm{C}$ " da voçoroca, área que se encontra em estágio inicial de regeneração, foi o ambiente que apresentou o maior número de espécies de formigas capturadas. Resultados estes corroborados por Schmidt e Diehl (2008) que, estudando o comportamento da comunidade de formigas em áreas sob diferentes usos do solo, no sul do Brasil, apresentaram como resultado uma maior riqueza de formigas capturadas em áreas selecionadas em estágio inicial de regeneração.

Cerne, Lavras, v. 19, n. 4, p. 661-668, out./dez. 2013 
Tabela 1 - Lista de espécies de formigas capturadas na área de voçoroca (braços: "A", "B", "C"), pastagem e mata em Itumirim-MG, 2010.

Table 1 - List of ant species captured in gully erosion (sectors " $A$ ", " B", "C") grassland and forest in Itumirim-MG, 2010.

\begin{tabular}{|c|c|c|c|c|c|}
\hline Família Formicidae & Mata & \multicolumn{2}{|c|}{ A B } & \multirow[t]{2}{*}{ C } & Pastagem \\
\hline Subfamília Dolichoderinae & & & & & \\
\hline Dolichoderini & & & & & \\
\hline Dorymyrmex sp. 1 (Mayr, 1866) & & & & $\mathrm{x}$ & \\
\hline Linepithema sp. 1 (Mayr, 1866) & & $\mathrm{x}$ & $\mathrm{x}$ & $\mathrm{x}$ & $\mathrm{x}$ \\
\hline Linepithema sp. 2 & & & & $x$ & \\
\hline Tapinomini & & & & & \\
\hline Tapinoma sp. 1 (Förster, 1850) & & & & $x$ & $\mathrm{x}$ \\
\hline Tapinoma sp. 2 & $\mathrm{x}$ & & & $\mathrm{x}$ & \\
\hline
\end{tabular}

\begin{tabular}{lc}
\hline Subfamília Ecitoninae & \\
\hline Ecitonini & \\
Eciton sp. 1 & $\mathrm{x}$ \\
Eciton sp. 2 & $\mathrm{x}$ \\
\hline Subfamília Ectatomminae & \\
\hline
\end{tabular}

\begin{tabular}{|c|c|c|c|c|c|}
\hline \multicolumn{6}{|l|}{ Ectatommini } \\
\hline Ectatomma edentatum (Roger, 1863) & & $\mathrm{x}$ & & & \\
\hline Ectatoma sp. 2 & $\mathrm{x}$ & & $\mathrm{x}$ & $\mathrm{x}$ & $\mathrm{x}$ \\
\hline Ectatoma sp. 3 & & $\mathrm{x}$ & & $\mathrm{x}$ & \\
\hline $\begin{array}{l}\text { Gnamptogenys striatula } \\
\text { (Radoszkowsky, 1884) }\end{array}$ & $\mathrm{x}$ & & $\mathrm{x}$ & & \\
\hline Gnampthogenys sp. 1 (Roger, 1863) & & & & & $\mathrm{x}$ \\
\hline Gnampthogenys sp. 2 & & & $\mathrm{x}$ & & \\
\hline Gnampthogenys sp. 3 & & & & & $\mathrm{x}$ \\
\hline Gnampthogenys sp. 4 & $\mathrm{x}$ & & & & \\
\hline Gnampthogenys sp. 5 & & & $\mathrm{x}$ & & \\
\hline \multicolumn{6}{|l|}{ Subfamília Formicinae } \\
\hline \multicolumn{6}{|l|}{ Brachymyrmecini } \\
\hline Brachymyrmex sp. 1 (Mayr, 1868) & & & & $\mathrm{x}$ & $\mathrm{x}$ \\
\hline Brachymyrmex sp. 2 & & & & $\mathrm{x}$ & \\
\hline Brachymyrmex sp. 3 & & & $\mathrm{x}$ & & \\
\hline Brachymyrmex sp. 4 & & & & $\mathrm{x}$ & \\
\hline \multicolumn{6}{|l|}{ Camponotini } \\
\hline Camponotus sp. 1 (Mayr, 1861) & & & $\mathrm{x}$ & $\mathrm{x}$ & $\mathrm{x}$ \\
\hline Camponotus sp. 2 & & $\mathrm{x}$ & $\mathrm{x}$ & & $\mathrm{x}$ \\
\hline
\end{tabular}

Continua...

To be continued...
Tabela 1 - Continuação...

Table 1 - Continued...

\begin{tabular}{|c|c|c|c|c|c|}
\hline Família Formicidae & Mata & $\mathrm{A}$ & $\mathrm{B}$ & $\mathrm{C}$ & Pastagem \\
\hline \multicolumn{6}{|l|}{ Subfamília Formicinae } \\
\hline Camponotus sp. 3 & $\mathrm{x}$ & & $\mathrm{x}$ & $\mathrm{x}$ & \\
\hline Camponotus sp. 4 & $\mathrm{x}$ & & & & \\
\hline Camponotus sp. 5 & & & & $\mathrm{x}$ & $\mathrm{x}$ \\
\hline \multicolumn{6}{|l|}{ Camponotus sp. 6} \\
\hline Camponotus sp. 7 & & & $\mathrm{x}$ & $\mathrm{x}$ & $\mathrm{x}$ \\
\hline $\begin{array}{l}\text { Camponotus rufipes (Fabricius, } \\
1775 \text { ) }\end{array}$ & & $\mathrm{x}$ & $\mathrm{x}$ & $\mathrm{x}$ & \\
\hline Camponotus sp. 9 & & & & $\mathrm{x}$ & \\
\hline Camponotus sp. 10 & & & & $\mathrm{x}$ & $\mathrm{x}$ \\
\hline Camponotus sp. 11 & & & & $\mathrm{x}$ & \\
\hline Camponotus sp. 12 & & & $\mathrm{x}$ & & \\
\hline \multicolumn{6}{|l|}{ Myrmelachistini } \\
\hline Myrmelachista sp. 1 (Roger, 1863) & & $\mathrm{x}$ & & & \\
\hline Plagiolepidini & & & & & \\
\hline
\end{tabular}

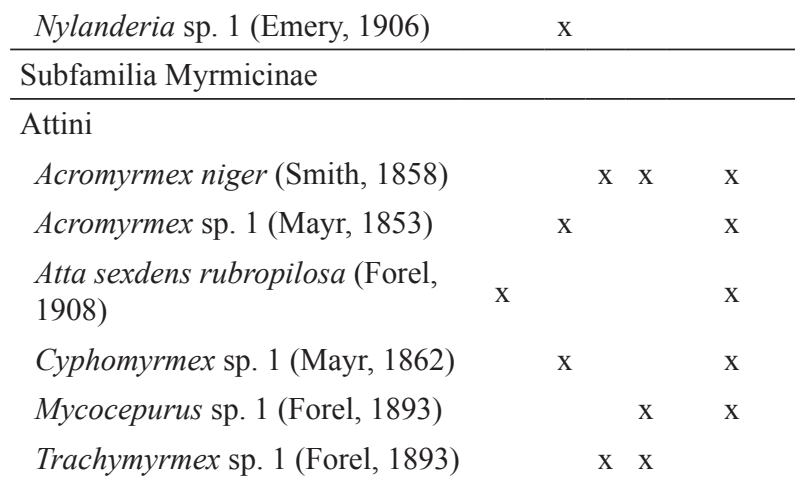

Blepharidattini

Wasmannia sp. 1 (Forel, 1893) x

Wasmannia sp. 2

Cephalotini

Cephalotes sp. 1 (Latreille, 1802) x

Crematogastrini

Crematogaster sp. 1 (Lund, 1931) x $\quad$ x

Crematogaster sp.2 $\mathrm{x}$

Crematogaster sp.3 3

Dacetonini

Strumygenis sp.1 $\quad \mathrm{x}$

Continua...

To be continued...

Cerne, Lavras, v. 19, n. 4, p. 661-668, out./dez. 2013 
Tabela 1 - Continuação...

Table 1 - Continued...

\begin{tabular}{|c|c|c|c|c|c|}
\hline Família Formicidae & Mata & A $\mathrm{I}$ & $\mathrm{B}$ & $\mathrm{C}$ & Pastagem \\
\hline \multicolumn{6}{|l|}{ Subfamilia Myrmicinae } \\
\hline \multicolumn{6}{|l|}{ Myrmicini } \\
\hline Hylomyrma sp.1 (Forel, 1912) & & & & $\mathrm{x}$ & \\
\hline \multicolumn{6}{|l|}{ Pheidolini } \\
\hline Pheidole sp. 1 (Westwood, 1839) & $\mathrm{x}$ & $\mathrm{x}$ & & & \\
\hline Pheidole sp. 2 & & & & $\mathrm{x}$ & $\mathrm{x}$ \\
\hline Pheidole sp. 3 & & & & $\mathrm{x}$ & $\mathrm{x}$ \\
\hline Pheidole sp. 4 & $\mathrm{x}$ & & $\mathrm{x}$ & & \\
\hline Pheidole sp. 5 & & & & $\mathrm{x}$ & \\
\hline Pheidole sp. 6 & $\mathrm{x}$ & & & & \\
\hline Pheidole sp. 7 & $\mathrm{x}$ & & & & \\
\hline Pheidole sp. 8 & & & & $\mathrm{x}$ & $\mathrm{x}$ \\
\hline Pheidole sp. 9 & & $\mathrm{x} \quad 2$ & $\mathrm{x}$ & & $\mathrm{x}$ \\
\hline Pheidole sp. 10 & $\mathrm{x}$ & & & & $\mathrm{x}$ \\
\hline Pheidole sp. 11 & & $\mathrm{x} \quad 2$ & $\mathrm{x}$ & & $\mathrm{x}$ \\
\hline Pheidole sp. 12 & & $\mathrm{x}$ & & & \\
\hline Pheidole sp. 13 & & & & & $\mathrm{x}$ \\
\hline Pheidole sp. 14 & & & & & $\mathrm{x}$ \\
\hline Pheidole sp. 15 & & $\mathrm{x}$ & & & \\
\hline Pheidole sp. 16 & & & & & $\mathrm{x}$ \\
\hline Solenopsidini & & & & & $\mathrm{x}$ \\
\hline Solenopsis sp. 1 (Westwood, 1840) & & $\mathrm{x}$ & & $\mathrm{x}$ & \\
\hline Solenopsis sp. 2 & $\mathrm{x}$ & $\mathrm{x}$ & & $\mathrm{x}$ & \\
\hline Solenopsis sp. 3 & & & $\mathrm{x}$ & & \\
\hline \multicolumn{6}{|l|}{ Subfamília Ponerinae } \\
\hline $\begin{array}{l}\text { Ponerineae sp. } 1 \text { (Donisthorpe, } \\
\text { 1943) }\end{array}$ & $\mathrm{x}$ & & & & \\
\hline \multicolumn{6}{|l|}{ Ponerini } \\
\hline Anochetus sp. 1 (Mayr, 1861) & & $\mathrm{x}$ & & & \\
\hline $\begin{array}{l}\text { Odontomachus sp. } 1 \text { (Latreille, } \\
\text { 1804) }\end{array}$ & & $\mathrm{x}$ & & & \\
\hline Pachycondyla striata (Smith, 1858) & $\mathrm{x}$ & $\mathrm{x} \quad 2$ & $\mathrm{x}$ & $\mathrm{x}$ & \\
\hline Pachycondyla verenae (Forel, 1922) & & $\mathrm{x} \quad 2$ & $\mathrm{x}$ & $\mathrm{x}$ & $\mathrm{x}$ \\
\hline \multicolumn{6}{|l|}{ Subfamília Pseudomyrmicinae } \\
\hline \multicolumn{6}{|l|}{ Pseudomyrmecini } \\
\hline $\begin{array}{l}\text { Pseudomyrmex gracilis } \\
\text { (Fabricius, 1804) }\end{array}$ & & $\mathrm{x}$ & & & $\mathrm{x}$ \\
\hline Pseudomyrmex sp. 1 & & $\mathrm{x}$ & & & \\
\hline
\end{tabular}

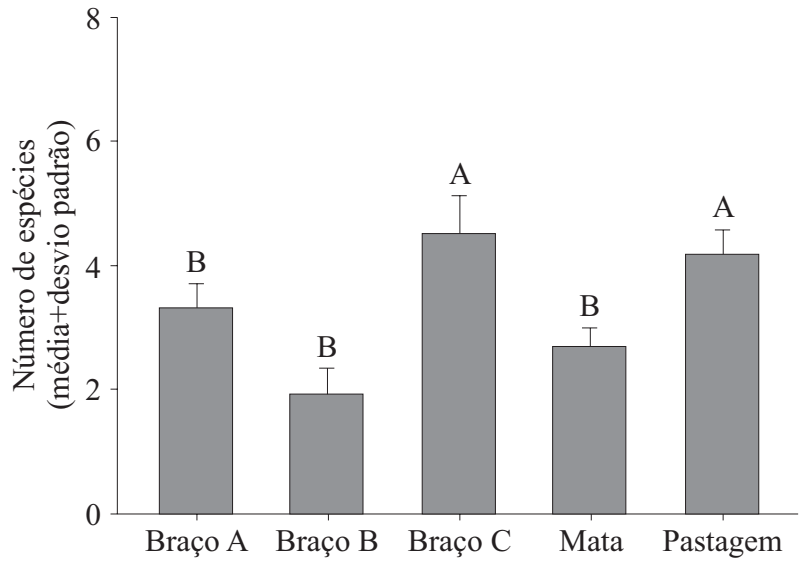

Figura 3 - Médias do número de espécies de formigas capturadas por "pitfall" em uma voçoroca e no seu entorno no município de Itumirim-MG. (Médias com mesma letra não se diferem estatisticamente pelo teste de Scott-Knott 5\%).

Figure 3 - Means number of ant species caught per pitfall in a gully erosion and its surroundings in the city of Itumirim- $M G$. (Means with same letter do not differ statistically by Scott-Knott test 5\%).

Resultados semelhantes também foram obtidos em Sidney na Austrália, que levaram em consideração variáveis de complexidade ambiental, como cobertura da vegetação herbácea, arbustiva e arbórea e demonstraram que a diversidade de formigas em áreas com baixa complexidade foi maior do que em áreas com alta complexidade ambiental (LASSAU; HOCHULI, 2004). A perturbação do hábitat pode, inicialmente, aumentar o número de espécies em uma área (ARMBRECHT et al., 2004; CORRÊA et al., 2006). As áreas perturbadas podem ser preferidas e "visitadas" pelas formigas, em razão da sua baixa complexidade e competitividade. São áreas abertas, com maior intensidade de luz sobre o solo, onde o calor favorece a disponibilidade de energia para o forrageamento e a reprodução de algumas espécies de formigas (HÖLLDOBLER; WILSON, 1990).

A não relação entre o tempo de recuperação e a riqueza de formigas é comum (COELHO et al., 2009; NICHOLS; NICHOLS, 2003; OTTONETTI et al., 2006), e pode-se inferir que, neste estudo, mesmo com o aumento da disponibilidade dos recursos e melhoria nas condições ambientais ao longo da recuperação, as espécies ainda podem competir pelos mesmos o que não leva a um aumento do número de espécies (CORRÊA et al., 2006). Outras possíveis explicações são que o remanescente florestal e a pastagem são muito próximos e a colonização

Cerne, Lavras, v. 19, n. 4, p. 661-668, out./dez. 2013 
por espécies de formigas pode ser rápida ou a recuperação, com o incremento na riqueza e abundância de espécies vegetais, não é suficiente para que as formigas percebam essa mudança como melhores condições e recursos para seu estabelecimento nas áreas. Outra possível explicação, é que os ambientes em estágio inicial de regeneração são invadidos por espécies generalistas, o que torna este parâmetro, riqueza de espécies, inadequado para avaliação da recuperação, como já discutido por outros autores (RIBAS et al., 2012). $\mathrm{O}$ número de espécies de formigas apresenta respostas variáveis a diferentes situações de acordo com o habitat, a intensidade da perturbação ou tempo decorrido desde a perturbação (HOFFMANN; ANDERSEN, 2003).

$\mathrm{Na}$ ordenação dos ambientes em função das espécies pela "Nonmetric Multidimensional Scaling" (NMDS), o gráfico revela um agrupamento referente ao ambiente mata, diferenciando-o dos demais ambientes (Figura 4).

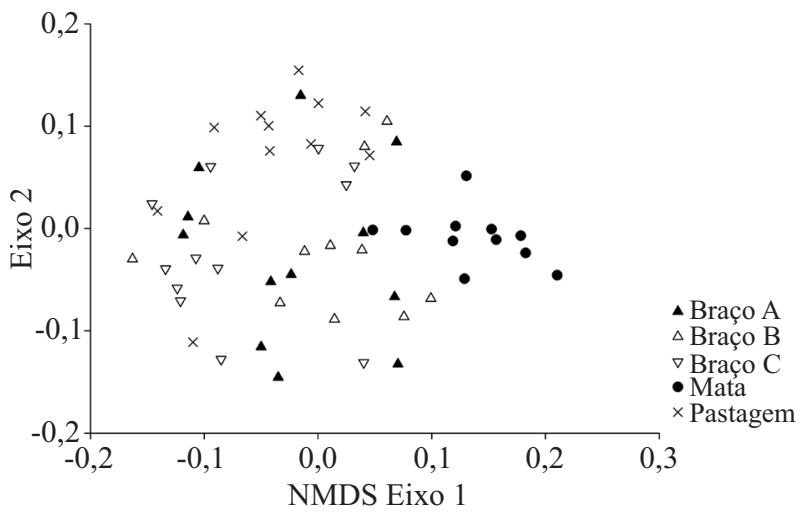

Figura 4 - Ordenação NMDS, por meio do índice Raup-Crick, das "pitfall" dos cinco ambientes baseada na presença/ausência das espécies de formigas em ambiente da voçoroca (braço A, braço B, braço C), mata e pastagem, Itumirim-MG.

Figure 4-NMDS ordination through the Raup-Crick index of the pitfall of the five environments based on the presence / absence of the species of ants in a gully erosion (setor A, sector B, sector $C)$, forest and grassland in Itumirim-MG.

$\mathrm{Na}$ avaliação da similaridade na composição de espécies de formigas, o teste ANOSIM apresentou resultados significativos entre os ambientes mata $\mathrm{x}$ voçoroca, e pastagem $\mathrm{x}$ voçoroca (Tabela 2). Os valores de correlação indicam uma maior dissimilaridade na composição de espécies de formigas do interior da voçoroca com o ambiente mata, indicando uma possível colonização da voçoroca por espécies de formigas oriundas da pastagem.
Tabela 2 - Dissimilaridade (valor de R) obtidos através da análise de similaridade (ANOSIM) comparando a composição das espécies de formigas dos ambientes no interior da voçoroca (braço A, braço B, braço C), mata e pastagem, Itumirim-MG, 2010.

Table 2 -Dissimilarity ( $R$ value) obtained by similarity analysis (ANOSIM) comparing the composition of ant species of environments within the gully erosion (sector A, sector B, sector C), forest and pasture, Itumirim-MG, 2010.

\begin{tabular}{lccccc}
\hline \multicolumn{5}{c}{ Valores de R } \\
\hline & Mata & Braço A & Braço B & Braço C & Pastagem \\
\hline Mata & - & & & & \\
Braço A & $0,61^{*}$ & - & & & \\
Braço B & $0,51^{*}$ & $0,02^{\text {ns }}$ & - & & \\
Braço C & $0,71^{*}$ & $0,05^{\text {ns }}$ & $0,05^{\text {ns }}$ & - & \\
Pastagem & $0,74^{*}$ & $0,17^{*}$ & $0,25^{*}$ & $0,22^{*}$ & - \\
\hline
\end{tabular}

*valores significativos $(\mathrm{p}<0,05)$

$\mathrm{ns}=$ valores não significativos

Os impactos ambientais geralmente resultam em uma mudança significativa na composição de espécies e, possivelmente, uma substituição de espécies nas comunidades de formigas, tanto em ambientes simplificados como em ambientes em regeneração (HOFFMANN, 2000; LASSAU; HOCHULI, 2004; UEHARA-PRADO et al., 2009; UNDERWOOD; FISHER, 2006). Compreende-se que locais degradados, geralmente, são colonizados por espécies oportunistas ou espécies generalistas, que podem obter vantagens com as mudanças dos recursos, alterando as condições de sobrevivência nos ambiente em questão (HOFFMANN; ANDERSEN, 2003).

\section{CONCLUSÕES}

No processo de recuperação de uma voçoroca com diferentes estágios de regeneração, o ambiente mais degradado e em estágio inicial de regeneração, apresentou maior riqueza de espécies de formigas.

A composição de espécies de formigas na área estudada revelou mudanças das espécies capturadas nos ambientes em recuperação, mostrando que, possivelmente, a colonização de formigas no interior da voçoroca ocorreu, principalmente, por espécies oriundas da área de pastagem.

Os parâmetros das comunidades de formigas, riqueza e composição de espécies, foram influenciados pela regeneração da área, indicando que as formigas podem ser usadas como bioindicadoras de recuperação de voçorocas.

Cerne, Lavras, v. 19, n. 4, p. 661-668, out./dez. 2013 


\section{AGRADECIMENTOS}

Ao CNPq, pela bolsa de estudo concedida ao primeiro autor deste trabalho.

\section{REFERÊNCIAS}

ALONSO, L. E.; AGOSTI, D. Biodiversity studies, monitoring and ants: an overview. In: AGOSTI, D. et al. (Ed.). Ants: standard methods for measuring and monitoring biodiversity. Washington: Smithsonian Institution, 2000. p. 1-8.

ANTUNES, F. Z. Caracterização climática do Estado de Minas Gerais. Informe Agropecuário, Belo Horizonte, v. 12, n. 138 , p. 9-13, jun. 1986.

ARMBRECHT, I.; PERFECTO, I.; VANDERMEER, J. Enigmatic biodiversity correlations: and diversity responds to diverse resources. Science, New York, v. 304, p. 284-286, 2004.

BENNETT, J. M.; KUTT, A. S.; JOHNSON, C. N.; ROBSON, S. K. A. Ants as indicators for vertebrate fauna at a local scale: an assessment of cross-taxa surrogacy in a disturbed matrix.

Biodiversity Conservation, New York, v. 18, n. 13, p. 34073419, 2009.

BOLTON, B. A. Identification guide to the ant genera of the world. Cambridge: Harvard University, 1994.

BRUYN, A. L. de. Ants as bioindicators of soil function in rural environments. Agriculture, Ecosystems and Environment, Amsterdam, v. 74, n. 1/3, p. 425-441, June 1999.

CARVALHO, D. A. Flora fanerogâmica de campos rupestres da Serra da Bocaina, Minas Gerais: caracterização e lista de espécies. Ciência e Prática, Lavras, v. 16, n. 1, p. 97-122, jan./jun. 1992.

CLARKE, K. R.; WARWICK, R. M. Change in marine communities: an approach to statistical analysis and interpretation. Plymouth: Plymouth Marine Laboratory, 2001. $172 \mathrm{p}$.

COELHO, M. S.; FERNANDES, G. W.; SANTOS, J. C.; DELABIE, J. H. C. Ants (Hymenoptera: Formicidae) as bioindicators of land in a Brazilian Atlantic Forest Fragment. Sociobiology, Chicago, v. 54, p. 51-63, 2009.
CORRÊA, M. M.; FERNANDEZ, W. D.; LEAL, I.

R. Diversidade de formigas epigéicas (Hymenoptera: Formicidae) em Capões do Pantanal Sul Matogrossense: relações entre riqueza de espécies e complexidade estrutural da área. Neotropical Entomology, Londrina, v. 35, p. 724$730,2006$.

HAMMER, O.; HARPER, D. A. T.; RIAN, P. D. Past: palaeonthological statistics software package for education and data analysis. Version 1.37. Disponível em: $<$ http://palaeoelectronica.org/2001_1/past/issue1_01.htm>. Acesso em: 12 nov. 2010.

HOFFMANN, B. D. Changes in ant species composition and community organisation along grazing gradients in semiarid rangelands of the Northern Territory. Australian Rangeland Journal, Cottesloe, v. 22, n. 2, p. 171-189, 2000.

HOFFMANN, B. D.; ANDERSEN, A. N. Responses of ants to disturbance in Australia, with particular reference to functional groups. Austral Ecology, Carlton, v. 28, n. 4, p. 444-464, 2003.

HÖLLDOBLER, B.; WILSON, E. O. The ants. Cambridge: Harvard University, 1990. 732 p.

LASSAU, S. A.; HOCHULI, D. F. Effects of habitat complexity on ant assemblages. Ecography, Copenhagen, v. 27, n. 2, p. 157-164, 2004.

LEAL, I. R. Dispersão de sementes por formigas na caatinga. In: LEAL, I. R.; TABARELLI, M.; SILVA, J. M. (Ed.).

Ecologia e conservação da caatinga. Recife: UFPE, 2003. p. 435-460.

LOSCHI, R. A. Interações espécie-ambiente na colonização de uma voçoroca em Itumirim, Minas Gerais. 2009. 67 p.

Dissertação (Mestrado em Ciências Florestais) - Universidade Federal de Lavras, Lavras, 2009.

MALEQUE, M. A.; MAETO, K.; ISHII, H. T. Arthropods as bioindicators of sustainable forest management, with a focus on plantation forests. Applied Entomology and Zoology, Tokyo, v. 44, n. 1, p. 1-11, 2009.

MCGEOCH, M. A. The selection, testing and application of terrestrial insects as bioindicators. Biological Review, Cambridge, v. 73, n. 1, p. 181-201, 1998.

Cerne, Lavras, v. 19, n. 4, p. 661-668, out./dez. 2013 
NICHOLS, O. G.; NICHOLS, F. M. Long-term trends in faunal recolonization after bauxite mining in the Jarrah Forest of Southwestern Australia. Restoration Ecology, Malden, v. 11, p. 261-272, 2003.

NIEMI, G. J.; MCDONALD, M. E. Application of ecological indicators. Annual Review of Ecology, Evolution, and

Systematics, Palo Alto, v. 35, p. 89-111, June 2004.

OTTONETTI, L.; TUCCI, L.; SANTINI, G. Recolonization patterns of ants in a rehabilitated lignite mine in central Italy: potential for the use of Mediterranean Ants as indicators of restoration processes. Restoration Ecology, Malden, v. 14, n. 1, p. 60-66, 2006.

R DEVELOPMENT CORE TEAM. R: a language and environment for statistical computing. Vienna: Computing RFS, 2005. Disponível em: <http://www.R-project.org>. Acesso em: 21 out. 2010.
RIBAS, C. R.; CAMPOS, R. B. F.; SCHMIDT, F. A.; SOLAR, R. R. C. Ants as indicators in Brazil: a review with suggestions to improve the use of ants in environmental monitoring programs. Psyche, Cambridge, v. 1, p. 1-23, 2012.

SCHMIDT, F. A.; DIEHL, E. What is the effect of soil use on ant communities? Neotropical Entomology, Londrina, v. 37 , n. 4, p. 381-388, 2008 .

UEHARA-PRADO, M.; FERNANDES, J. O.; MOURABELLO, A.; MACHADO, G.; SANTOS, A. J.; VAZ-DEMELLO, F. Z.; FREITAS, A. V. L. Selecting terrestrial arthropods as indicators of small-scale disturbance: a first approach in the Brazilian Atlantic Forest. Biological Conservation, Cambridge, v. 142, n. 6, p. 1220-1228, 2009.

UNDERWOOD, E. C.; FISHER, B. L. The role of ants in conservation monitoring: if, when, and how. Biological Conservation, Cambridge, v. 132, n. 2, p. 166-182, 2006.

Recebido: 29 de março de 2011; aceito: 26 de junho de 2013.

Cerne, Lavras, v. 19, n. 4, p. 661-668, out./dez. 2013 